\title{
Laser Fabrication of Polymer Ferroelectric Nanostructures for Non-volatile Organic Memory Devices
}

Daniel E. Martínez-Tong ${ }^{1, \text {,* }}$, Álvaro Rodríguez-Rodríguez ${ }^{1}$, Aurora Nogales ${ }^{1}$, Mari Cruz García-Gutiérrez ${ }^{1}$, Francesc Pérez-Murano ${ }^{2}$, Jordi Llobet ${ }^{2}$, Tiberio A. Ezquerra ${ }^{1}$ and Esther Rebollar ${ }^{3, *}$

${ }^{1}$ Instituto de Estructura de la Materia (IEM-CSIC). C/ Serrano 121. Madrid, 28006. Spain

${ }^{2}$ Instituto de Microelectrónica de Barcelona IMB-CNM (CSIC), Campus UAB 08193, Cerdanyola del Vallès (Bellaterra) Barcelona, Spain

${ }^{3}$ Instituto de Química Física Rocasolano (IQFR-CSIC). C/ Serrano 119. Madrid, 28006. Spain

*Corresponding authors: danmarti@ulb.ac.be

e.rebollar@iqfr.csic.es

${ }^{\dagger}$ Present address: Département de Physique, Faculté des Sciences, Université libre de Bruxelles (ULB), Boulevard du Triomphe, 1050 Brussels, Belgium

\begin{abstract}
Polymer ferroelectric laser induced periodic surface structures (LIPSS) have been prepared on ferroelectric thin films of a poly(vinylidene fluoride- trifluoro ethylene) copolymer. Although not absorbing light at the laser wavelength, LIPSS on the copolymer can be obtained by forming a bilayer with other light absorbing polymer. The ferroelectric nature of the structured bilayer was proven by piezoresponse force microscopy measurements. Ferroelectric hysteresis was found on both bilayer and laser structured bilayer. We show that it is possible to write ferroelectric information at the nanoscale. The laser structured ferroelectric bilayer showed an increase in the information storage density of an order of magnitude in comparison to the original bilayer.
\end{abstract}

Keywords: Laser Induced Periodic Surface Structures; Non-volatile Ferroelectric Memories; Piezoresponse Force Microscopy; Polymer bilayers; Nanogratings 


\section{Introduction}

In recent years, research on ferroelectric polymers has been focused towards the development of functional prototypes for all-organic electronics. Functional devices, such as diodes $^{1-2}$ and transistors ${ }^{3-5}$, have been fabricated in a layer-by-layer approach by combining thin films of ferroelectric polymers with other materials like semiconducting polymers ${ }^{4}$ and inorganic alloys ${ }^{6}$. Moreover, the use of nanopatterned surfaces in these devices has shown to improve the ferroelectric response of the polymers ${ }^{7-8}$, enhance the storage information density by controlling molecular architecture ${ }^{9-12}$, and also allow the possibility of preparing 3-dimensional nanostructures, such as multiple-gate transistors $^{13-15}$.

Surface nanostructuring of polymers can be accomplished with a great variety of procedures, such as nanoimprint lithography (NIL) ${ }^{16-17}$, photolithography ${ }^{18}$, laser interference $^{19}$ and optical near-fields ${ }^{20-21}$. Also, laser induced periodic surface structures (LIPSS) can be prepared on polymer thin films ${ }^{22-23}$. LIPSS can appear after irradiation of solid surfaces by intense laser pulses, as a result of the interference between the incoming and the surface-scattered waves of the laser. This causes an heterogeneous energy intensity distribution, which together with a feedback mechanism, results in a periodic enhancement of the irradiation intensity ${ }^{24}$. The final outcome is the appearance of spontaneous periodic surface nanostructures, with periodicities closely related to the wavelength of the irradiating laser ${ }^{24-26}$. Contrary to other techniques, LIPSS are prepared without the requirement of direct physical contact and clean environments. Also, it avoids problems like tearing during demolding and mold imperfections found in NIL structures ${ }^{4}$. 
The development of LIPSS on a polymer surface is related to both irradiation conditions and surface properties. Laser wavelength, fluence, pulse duration, polarization, angle of incidence and repetition rate, dictate the morphology of the resulting structures ${ }^{22-23,27-28}$ and allow the possibility of fabricating different geometrical arrays such as nanogratings and nanodots ${ }^{29}$. Typically in polymers, using nanosecond laser pulses, LIPSS are formed parallel to the polarization of the laser with a period $L$ described by $L=\lambda /(n-$ $\sin \theta)$ where $\lambda$ is the laser wavelength, $\mathrm{n}$ is the effective refraction index and $\theta$ is the incidence angle ${ }^{30}$. On the other hand, the polymer surface must fulfill certain conditions, such as light absorption at the specific wavelength and surface roughness on the order of the few nanometers. Previously, we have reported the formation of LIPSS on thin films of poly(ethylene terephthalate), poly(trimethylene terephthalate) and poly(bisphenol-A carbonate), using nanosecond (ns) pulses in the ultraviolet region ${ }^{22}$. However, in the case of the poly(vinylidene fluoride) (PVDF), no structuring could be achieved, due the poorly absorption of this polymer, at the same irradiation conditions, and its semicrystalline spherulitic morphology, which introduced further hindrances into the reorganization of the material as well as higher roughness ${ }^{22}$. The limitation of LIPSS method for nanostructuring non-absorbing polymers has been reported also by other authors $^{31}$. For this reason LIPSS formation was ruled out as a procedure to prepare ferroelectric nanostructures on PVDF and its copolymers.

In this work, we demonstrate two main effects. First, the possibility of LIPSS formation on non-absorbing polymer thin films using ns laser pulses. In order to overcome the absorption limitation, we have prepared bilayer polymer thin films in which the bottom layer absorbs light at the specific wavelength, while the top layer is formed by the nonabsorbing polymer of interest. We have selected the two functional polymer materials: P3HT as bottom absorbing layer, and P(VDF-TrFE) as non-absorbing top layer. 
Second, we proved that laser nanopatterning of the $\mathrm{P}(\mathrm{VDF}-\mathrm{TrFE})$ is achievable without compromising its ferroelectric response. We created nanostructures of $\mathrm{P}(\mathrm{VDF}-\mathrm{TrFE})$ whose storage information density increases in about one order of magnitude, in comparison to the non-structured bilayer. This validates the potential of our procedure in order to develop non-volatile organic memory devices.

\section{Experimental section}

2. 1. Materials. Poly(vinylidene fluoride-trifluoroethylene) $P(V D F-T r F E)$ was purchased from Piezotech S.A.S. Molar concentration of the random copolymer is 76:24 (VDF:TrFE content) and its molecular weight is $\mathrm{M}_{\mathrm{w}}=367 \mathrm{~kg} / \mathrm{mol}, \mathrm{M}_{\mathrm{w}} / \mathrm{M}_{\mathrm{n}}=$ 1.72 , melting temperature $=148 \pm 1{ }^{\circ} \mathrm{C}$. Poly $(3$-hexylthiophene $)(\mathrm{P} 3 \mathrm{HT})$ was purchased from Ossila (Batch M106, tradename lisicon ${ }^{\text {, }}$, molecular weight $\mathrm{M}_{\mathrm{w}}=34100 \mathrm{~g} / \mathrm{mol}$, $\left.\mathrm{M}_{\mathrm{w}} / \mathrm{M}_{\mathrm{n}}=1.7\right)$, melting temperature $=235 \pm 5^{\circ} \mathrm{C}$.

2.2. Polymer thin films. Polymers were used as received. Single-layer (SL) and Bilayer (BL) thin films were prepared by spin coating. For SL films, $0.2 \mathrm{~mL}$ of polymer solution was deposited on top of a $2 \times 2 \mathrm{~cm}^{2}$ square conductive silicon wafer (resistivity 0.001-0.005 $\Omega \mathrm{cm}$ ) and rotated at $2400 \mathrm{rpm}$ for 2 minutes. Specifically, $\mathrm{P}(\mathrm{VDF}-\mathrm{TrFE})$ was dissolved in 2-butanone $\left(\mathrm{C}_{4} \mathrm{H}_{8} \mathrm{O}\right)(5 \mathrm{mg} / \mathrm{mL})$, at $72^{\circ} \mathrm{C}$, under continuous stirring for 3 hours. P3HT was dissolved in chloroform $\left(\mathrm{CHCl}_{3}\right)(24 \mathrm{mg} / \mathrm{mL})$, at room temperature. Dissolution was achieved immediately within a few minutes.

The protocol for preparation of BL thin films is described next, and is similar to previous reports ${ }^{32-33}$. Solution concentrations were kept equal to the ones presented for SL films. First, a thin film of P3HT was spin coated on a $2 \times 2 \mathrm{~cm}^{2}$ conductive silicon wafer. Afterwards, the P(VDF-TrFE) solution was coated on top of the P3HT film. In both cases, a fixed rotation speed of $2400 \mathrm{rpm}$ for 2 minutes was used. In order to 
determine whether the 2-butanone had any effect on the P3HT, we performed a solubility test. The P3HT solution showed no coloration of the colorless 2-butanone after stirring, as previously reported in the literature ${ }^{32}$. This indicates the non-soluble character of the semiconducting polymer in the solvent.

2.3. Laser irradiation. Laser irradiation was carried out in ambient air, at normal incidence, with the linearly polarized laser beam of a Q-switched Nd:YAG laser (Lotis TII LS-2131M, pulse duration $\tau=8 \mathrm{ns,} \mathrm{full} \mathrm{width} \mathrm{at} \mathrm{half-maximum)} \mathrm{at} \mathrm{a} \mathrm{repetition} \mathrm{rate}$ of $10 \mathrm{~Hz}$. Second harmonic, at a wavelength $(\lambda)$ of $532 \mathrm{~nm}$, was used for the experiments. At this wavelength, P3HT has an absorption coefficient ${ }^{34-35}$ of $2 \times 10^{5} \mathrm{~cm}^{-1}$, while the PVDF based copolymer ${ }^{22}$ has one in the order of $10^{2} \mathrm{~cm}^{-1}$. This great difference among the absorption coefficients, at $\lambda=532 \mathrm{~nm}$, indicates that in the bilayer geometry, light will pass through the top $\mathrm{P}(\mathrm{VDF}-\mathrm{TrFE})$ layer without significant loss of energy, and reach the bottom P3HT film, where it will be absorbed. The fluences of irradiation were determined by measuring the laser energy in front of the sample with a joulemeter (Gentec-E, QE25SP-H-MB-D0) and calculating the area of the irradiated spots.

2.4. Atomic Force Microscopy (AFM). Morphology of the polymer SL and BL thin films was characterized by atomic force microscopy (AFM). A Multimode 8 AFM with a Nanoscope V controller (Bruker) was used under tapping mode with NSG-30 probes (NT-MDT). Square images with 512x512 pixels resolution were taken. Analysis of size and shape of the nanometric features was performed with the NanoScope Analysis 1.50 software (Bruker).

2.5. Piezoresponse Force Microscopy (PFM). PFM measurements were carried out by means of a Nanoscope V AFM (Bruker), in the piezoresponse mode. The topography 
and the ferroelectric signals were acquired simultaneously. The microscope was used in contact mode, with a low deflection set-point $(0.3 \mathrm{~V})$ in order to avoid damaging the samples. Conductive PtIr covered probes (SCM-PIC, Bruker, $k=0.2 \mathrm{~N} / \mathrm{m}$ ) were used. Through the PFM technique, hysteresis cycles were recorded applying a tip bias ramp from $-12 \mathrm{~V}$ to $12 \mathrm{~V}$ at a frequency of $0.1 \mathrm{~Hz}$ and collection of 1024 samples. Local poling was carried out using the tip as the top electrode. In every case, a DC bias ( \pm 12 V) was applied for 5 minutes. The control of points and lines drawn in the ferroelectric phase was made by means of the Point and Shoot protocol, available in the Nanoscope 8.15 software. The PFM out-of-plane signal was taken applying an AC voltage of $2 \mathrm{~V}$ at a frequency of $60 \mathrm{kHz}$ between sample and tip. Measured amplitudes are related to the local electromechanical response of the sample surface during application of an AC voltage.

2.6. Electrostatic Force Microscopy (EFM). Electrical measurements on the sample surface were also performed using the Nanoscope V AFM (Bruker). EFM images were taken using a double pass approach ${ }^{36-38}$. In this work, EFM experiments were carried out using Pt-Ir covered Si probes (SCM-PIT, Bruker, $k=4 \mathrm{~N} / \mathrm{m}$ ). Lift height was kept constant at $80 \mathrm{~nm}$ above each point on the surface, and the applied tip voltage was $+5 \mathrm{~V}$. Lift height was chosen in order to avoid effect of the topography.

2.7. Conductive Atomic Force Microscopy (C-AFM). Electrical measurements were performed with conductive probes (Pt-Ir covered Si cantilevers with a low spring constant, $k=0.2 \mathrm{Nm}^{-1}$, SCM-PIC by Bruker) in contact mode by measuring simultaneously both topography and electrical current images. In these measurements, the conducting probe makes contact with the sample, acting like a nanoelectrode, and maps a current image at a fixed bias of $-5 \mathrm{~V}$. The current was measured by a preamplifier. 
2.8. Cross sectional SEM. For the characterization of the bilayer thickness, crosssectional scanning electron microscope (SEM) images were performed with a focused ion beam (FIB, CrossBeam $1560 \mathrm{XB}$, Zeiss). For fabricating the cross-sections, the FIB was operated at $30 \mathrm{keV}$ for accelerating the gallium ion beam. In order to avoid damage to the polymer layers, small apertures were selected to provide a beam current of $50 \mathrm{pA}$. The sample was not covered with any metal layer during the FIB milling. The same equipment was used for obtaining the high resolution SEM images. The conditions for the imaging were $5 \mathrm{keV}, 30 \mu \mathrm{m}$ of aperture, a working distance of $5.1 \mathrm{~mm}$. The images were obtained using the InLens detector to enhance material contrast and resolution.

\section{Results and discussion}

\subsection{Polymer bilayers}

AFM topography images of the polymer thin films are shown in figure 1, before and after laser irradiation. Figures $1 \mathrm{a}$ and $1 \mathrm{~b}$ shows $5 \mathrm{x} 5 \mu \mathrm{m}^{2}$ AFM topography images of the as prepared P(VDF-TrFE) and P3HT SL films, respectively. In both cases, the AFM image shows a continuous surface without dewetting and/or agglomerates. The P(VDFTrFE) SL film exhibits a roughness of $1.0 \pm 0.5 \mathrm{~nm}$, while the P3HT SL film shows one of $4 \pm 1 \mathrm{~nm}$. These two polymers are semicrystalline, thus the roughness can be related to the presence of crystallites on the surface. Thickness of the films was measured by AFM. A scratch was made on the surface of the films and the step between the silicon surface and the polymer layer was quantified in different locations of several samples. The P(VDF-TrFE) SL thin film showed a thickness of $h_{\mathrm{P}(\mathrm{VDF}-\mathrm{TrFE})}=35 \pm 5 \mathrm{~nm}$. The thickness of the P3HT SL thin film was found to be $h_{\mathrm{P} 3 \mathrm{HT}}=140 \pm 10 \mathrm{~nm}$.

Figure 1c shows a $5 \times 5 \mathrm{~m}^{2}$ AFM topography image of the bilayer film. The resulting surface does not exhibit either signs of dewetting or mechanical instabilities as reported 
in other cases ${ }^{39-40} 41-42$. Also, it is worth noticing that our bilayers were prepared without requiring any external element to improve its mechanical stability in contrast to previous reports in the literature ${ }^{43}$. Roughness of the BL surface was quantified to be $1.2 \pm 0.5 \mathrm{~nm}$, comparable to the original P(VDF-TrFE) thin film. Step measurements of the bilayer film showed that the total thickness was $h_{\text {bilayer }}=200 \pm 50 \mathrm{~nm}$. In principle, this value is compatible with the addition of the individual thicknesses of every layer. However, in order to reach a more precise characterization of thickness and continuity of the upper polymer layer we have accomplished cross-sectional SEM measurements which are shown in figure 2. The two polymers appear as continuous layers of different electronic density in figure 2a. The thickness of the bilayer polymer film estimated by SEM gives values of $150 \pm 30 \mathrm{~nm}$ while upper and bottom layer exhibit thicknesses of $38 \pm 5 \mathrm{~nm}$ and $120 \pm 10 \mathrm{~nm}$ respectively. This result and the fact that the AFM topography image does not show signs of phase separation allows us concluding that the bilayer consists of two separate and continuous polymer layers one on top of the other in agreement with previous reports ${ }^{32-33}$. 

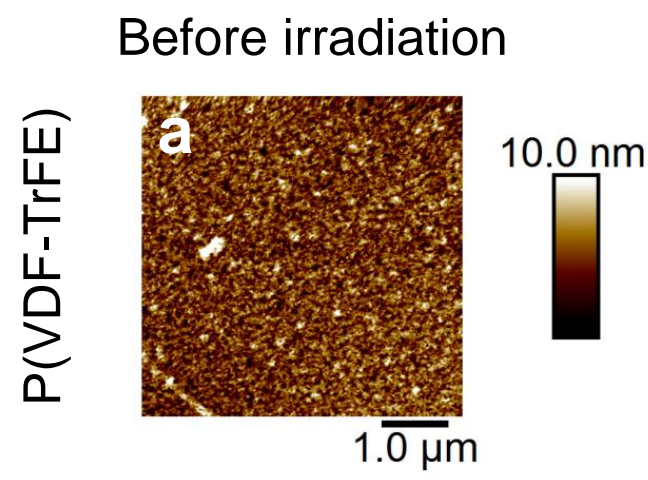

\section{After irradiation}
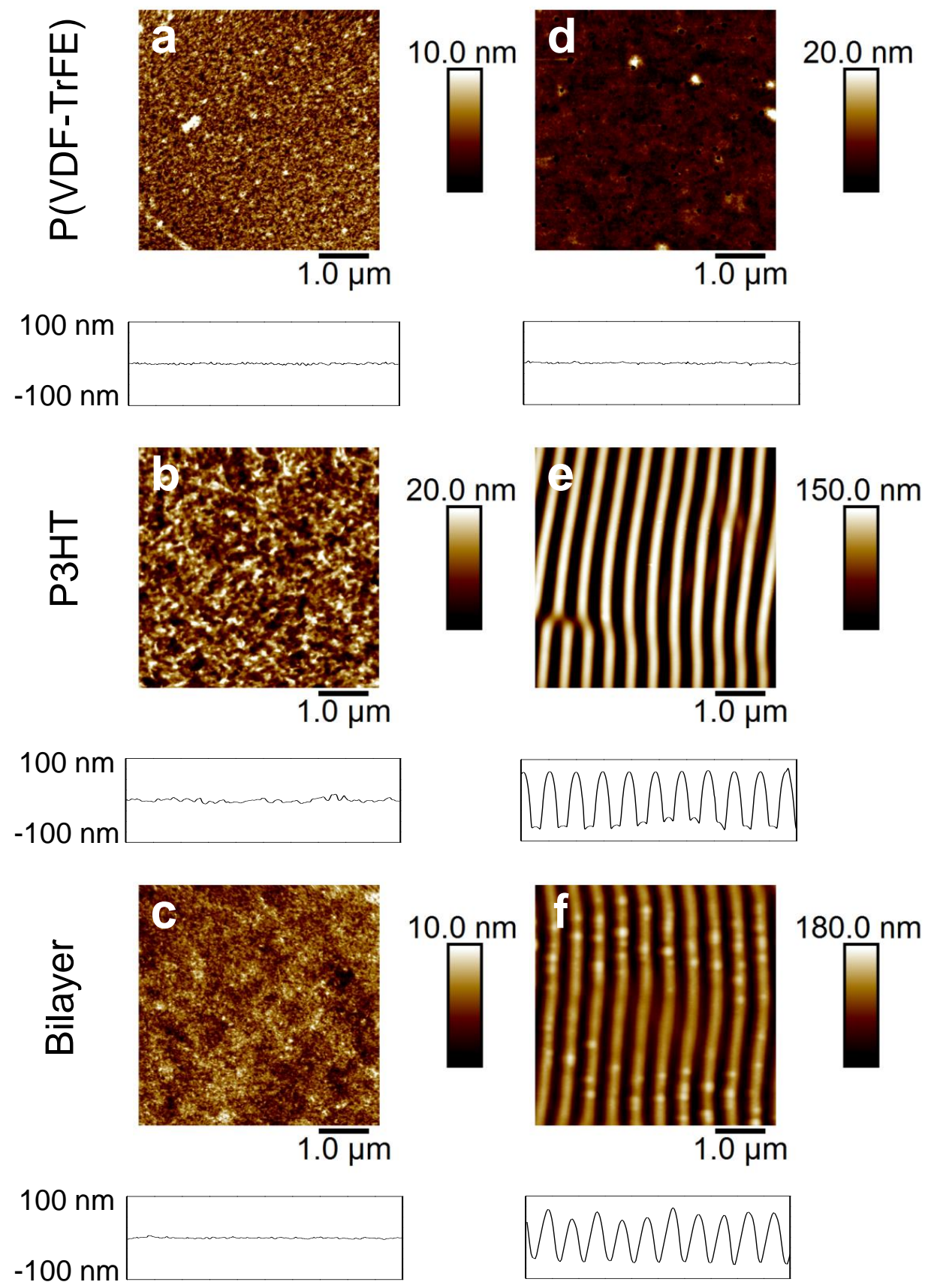

Figure 1. AFM topography images, before (left column) and after (right column) laser irradiation of single layer thin films of $\mathrm{P}(\mathrm{VDF}-\mathrm{TrFE})(\mathrm{a}, \mathrm{d})$ and $\mathrm{P} 3 \mathrm{HT}(\mathrm{b}, \mathrm{e})$ and of a $\mathrm{P}(\mathrm{VDF}-\mathrm{TrFE}) / \mathrm{P} 3 \mathrm{HT}$ thin bilayer film (c,f). LIPSS are formed on the P3HT thin film and on the bilayer. The height profile along a $5 \mu \mathrm{m}$ line is shown below every image. 

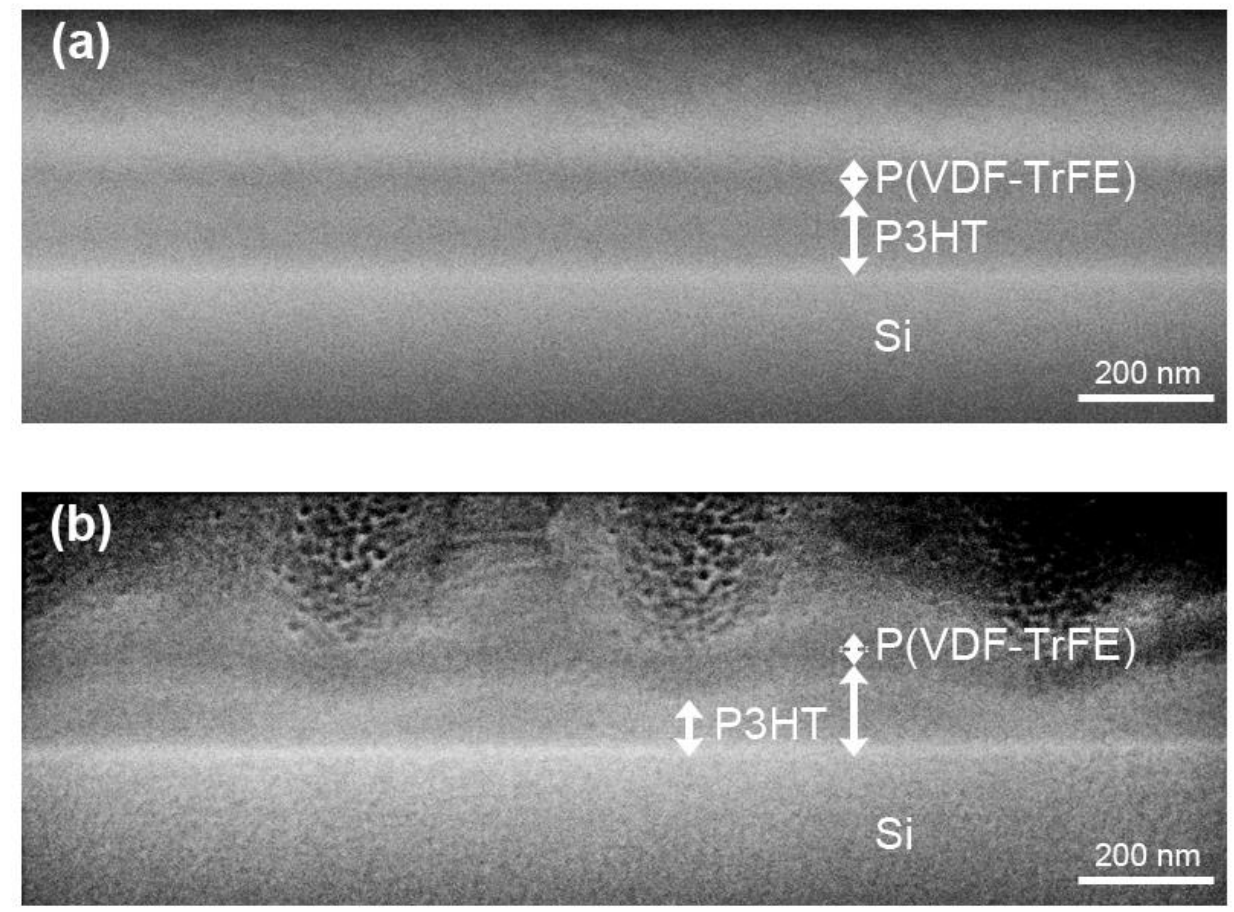

Figure 2. Cross-section SEM images of a $\mathrm{P}(\mathrm{VDF}-\mathrm{TrFE}) / \mathrm{P} 3 \mathrm{HT}$ thin bilayer film before (a) and after (b) irradiation.

\subsection{Laser Induced Periodic Surface Structures (LIPSS)}

Irradiation parameters (fluence and number of pulses) were chosen according to previously reported systematic experiments related to LIPSS formation on $\mathrm{P}_{3} \mathrm{HT}^{44}$. Right column of figure 1 shows $5 \times 5 \mathrm{~m}^{2}$ AFM topography images of SL and BL films after laser irradiation, at a fluence of $27 \mathrm{~mJ} \cdot \mathrm{cm}^{-2}$ and for 3600 pulses in every case. Figure 1d shows the result of laser irradiation on the P(VDF-TrFE) SL film. No development of nanostructures or any other surface features is observed while its roughness was found to remain around $1 \mathrm{~nm}$. The absence of surface structures on this copolymer can be related to its weak optical absorption ${ }^{22}$. Increasing the laser beam fluence did not allow the formation of any structuring until high fluence values, close to 
$700 \mathrm{~mJ} \cdot \mathrm{cm}^{-2}$, led to swelling of the film. Similar results have been previously reported for non-absorbing polymers ${ }^{45}$ such as $\mathrm{PVDF}^{46}$.

Comparison between figures $1 \mathrm{~b}$ and 1e evidences the morphology changes in the P3HT SL film. After laser irradiation, the formation of LIPSS is observed on its surface. The nanostructures consist of elongated ridges along a specific direction, giving raise to the development of nanogratings. The height of the nanostructures was found to be $100 \pm$ $20 \mathrm{~nm}$, while their period was about $460 \pm 10 \mathrm{~nm}$. As in our case the laser fluence is well below the ablation threshold, the structuring of the surface takes place without material removal ${ }^{23}$.

Figure 1f shows a $5 \times 5 \mathrm{~m}^{2}$ AFM topography image of the BL film, after laser irradiation. It can be seen that surface structuring took place and continuous linear nanostructures, with quantitative geometrical features similar to those of the P3HT, are observed. The continuity of the nanostructured upper polymer layer is corroborated by the cross-section SEM image shown in figure $2 b$. However, in order to rule out the possibility of discontinuity of the ferroelectric upper layer we have also performed both EFM and C-AFM measurements. The results are shown in figures S1 and S2 of the supplementary information. The EFM images reveal a fairly constant value of the electrostatic signal on either ridges or trench regions, with some contrast among them. The SEM images (figure 2b and figure S3, Supplementary Information) reveal that the trench regions of the nanostructure present some holes. Therefore, in a first approach, the contrast of the electrostatic field observed in the EFM images can be associated with this effect. Moreover, C-AFM images show that samples are non-conducting, confirming that the upper layer must be the $\mathrm{P}(\mathrm{VDF}-\mathrm{TrFE})$ film, homogeneously covering the P3HT. 
Since the P(VDF-TrFE) thin film did not show LIPSS at similar irradiation conditions (figure 1d) the development of the LIPSS on the bilayer must be associated with the P3HT bottom layer. As the P(VDF-TrFE) does not absorb light at the irradiating laser wavelength, the laser beam pass through the upper polymer layer without significantly altering its energy or polarization before reaching the P3HT bottom layer. Here, structuring takes place just as in the SL film heating the P3HT above its melting temperature in such a way that the polymer segments acquire enough mobility as to rearrange following the modulation imposed by the laser. Afterwards, heat dissipation from the P3HT allows cooling and fixing the nanostructure of the bottom layer while heating the $\mathrm{P}(\mathrm{VDF}-\mathrm{TrFE})$ on top. This heat transfer is expected to melt the ferroelectric polymer, gaining mobility and accommodating on the pattern of the bottom layer. The possibility of forming a nanostructured bilayer on which the top non-absorbing layer follows the bottom one is new and differs from previous reports in the literature. For example, Zhao and collaborators ${ }^{47}$ prepared surface patterns by laser irradiation on thin layers of poly(methyl methacrylate) (PMMA) on azobenzene liquid crystalline (LCP) polymer films. These authors found that the LCP single layer film reorganized following a grating-like structure after laser irradiation, using ns pulses. However, when dealing with the PMMA/LCP bilayer structure, the upper, non-absorbing layer of PMMA did not followed the LCP pattern but developed wavelike surface structures, whose periods did not relate to the wavelength of the laser pulse. Thus, by using this simple approach it is possible to form LIPSS on non-absorbing polymers.

In figure 1f, the LIPSS generated on the BL film show also the development of dropletlike structures on top. These are evidenced by the bright spots in the image and could be related to agglomeration of $\mathrm{P}(\mathrm{VDF}-\mathrm{TrFE})$ appearing during the melting and recrystallization process. These features, which are also visualized in the SEM images 
(figure S2, Supplementary Information), exhibit a round top with mean diameters ranging from $100-200 \mathrm{~nm}$ and heights from $20-50 \mathrm{~nm}$.

\subsection{Ferroelectric response of nanostructured bilayer films}

Figure 3 shows the PFM phase shifts as a function of an applied tip bias, for the prepared samples. As obtaining accurate electric field on films with complicated morphology can be misleading we have represented the phase shift as a function of the nominal applied voltage bias. In PFM, ferroelectric materials are characterized by hysteresis cycles with a $180^{\circ}$ shift of the PFM phase ${ }^{48}$. These changes are related to the flipping of polymer dipoles in the vertical direction, with respect to the sample surface (out-of-plane polarization), as in the charge displacement versus applied electric field measurements on thin film capacitors ${ }^{49}$. The value of the electric field where the shift of the PFM phase takes place is known as the coercive field $\left(\mathrm{E}_{\mathrm{c}}\right)$. As expected from its chemical composition, the P3HT sample (black line, figure 3a) shows no ferroelectric hysteresis, but simply a sudden transition from one state to other, consequence of the polarization of the tip and charge injection. Results for the P(VDF-TrFE) SL film are depicted in figure $3 \mathrm{a}$ with red lines. In this case, the ferroelectricity of the copolymer is evidenced by the $180^{\circ}$ PFM phase shift transition observed at non-zero field values. The coercive field can be estimated to be $\mathrm{E}_{\mathrm{c}}=(25 \pm 6) \mathrm{MV} / \mathrm{m}$, considering $h_{\mathrm{P}(\mathrm{VDF}-\mathrm{TrFE})}=35$ $\pm 5 \mathrm{~nm}$. This value is similar to those found in previous works for continuous films of this ferroelectric copolymer ${ }^{10,48}$, although smaller than previously reported values for thicker films ${ }^{50}$. In a first approach we can attribute this fact to differences in crystallinity depending on thickness. Finally, the non-irradiated bilayer film exhibits also a ferroelectric response, as evidenced from the hysteresis cycle shown by the blue curve in figure 3a. In this case, the coercive field was found to be around $E_{c}=(19 \pm 4) \mathrm{MV} / \mathrm{m}$. 


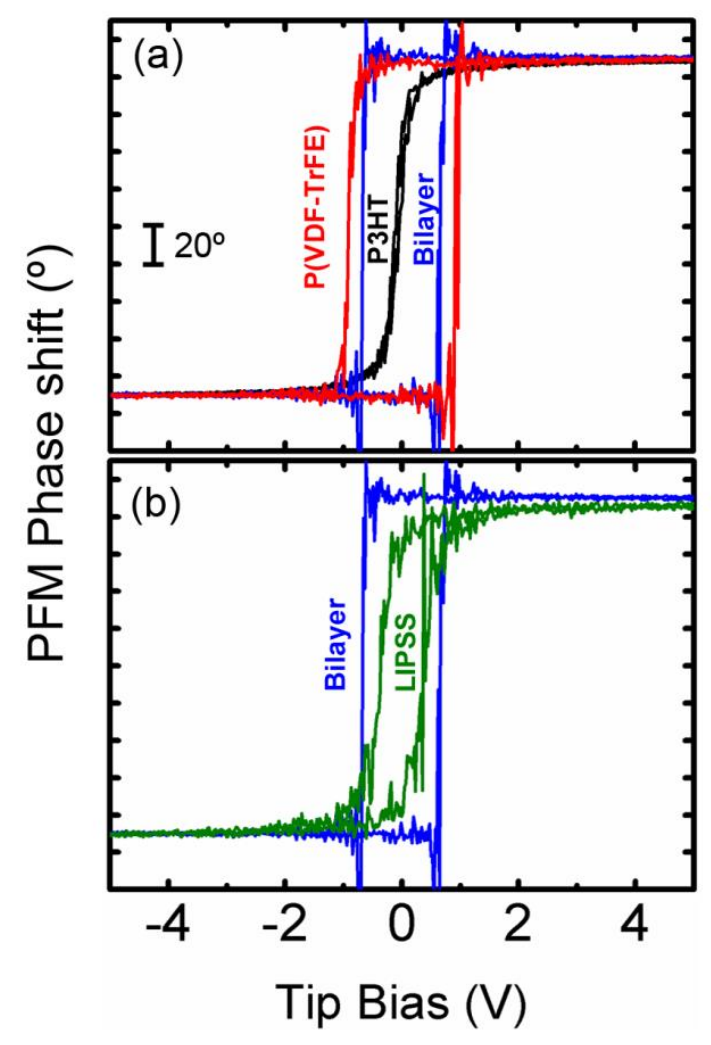

Figure 3. (a) PFM phase shift as a function of applied electric field for P3HT SL film (black), P(VDF-TrFE) SL film (red) and polymer bilayer (blue). Both P(VDF-TrFE) and bilayer film show ferroelectric hysteresis. (b) PFM phase shift as a function of applied bias for the LIPSS on the bilayer film (green) and continuous bilayer (blue) for comparison.

The ferroelectric response of the LIPSS BL film is shown in figure $3 \mathrm{~b}$ (green line). Data of the non-irradiated BL film is included for comparison. The presence of hysteresis indicates that the LIPSS have a ferroelectric response, with a coercive field close to $\mathrm{E}_{\mathrm{c}}=$ (10 \pm 4$) \mathrm{MV} / \mathrm{m}$. Hysteresis cycles were taken on several areas of the LIPSS BL and found to be equivalent. Evidence of ferroelectricity in the nanostructured layer further supports that the top layer follows the topography of the P3HT one. In addition, since the ferroelectricity of P(VDF-TrFE) depends strongly on its crystalline structure ${ }^{49}$, figure 3 suggests that laser irradiation did not inhibit the recrystallization of the P(VDFTrFE) copolymer. 


\subsection{Ferroelectric information storage on polymer bilayers}

Recently, PFM has also been used to store nanoscale information on ferroelectric organic films ${ }^{10,48,51-56}$, using the tip as a stylus to write the information by applying a bias above the coercive field. Reading is attained by the piezoelectric response of the sample, which gives different contrast between written and unwritten zones ${ }^{53}$ or by the difference in the electrical current that flows through the material ${ }^{2,4}$. Figure 4 shows $5 \mathrm{x} 1 \mu \mathrm{m}^{2}$ PFM images, where the ferroelectric state of the non-irradiated bilayer film, before and after poling for 5 minutes at a $+12 \mathrm{~V}$ bias, is observed. For the electric poling, the tip was fixed at a certain position on the bilayer surface, while the bias was applied. The magnitude of the physical contact between tip and surface was controlled by setting a low cantilever set-point.

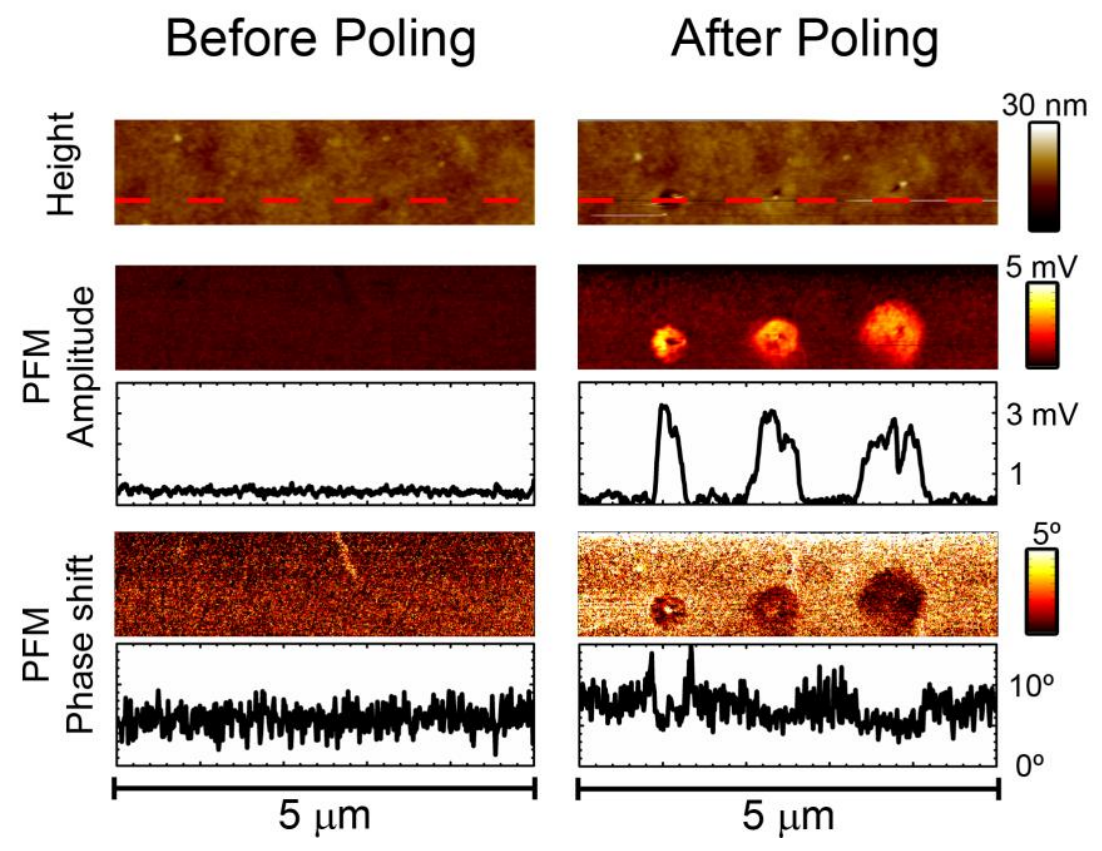

Figure 4. (Left column) Topography, PFM amplitude and PFM phase shift of the bilayer film before poling. (Right column) Topography, PFM amplitude and PFM phase shift of the bilayer film after poling at 3 points, for 5 minutes at $+12 \mathrm{~V}$. Panels third and fifth from top is the amplitude and phase shift across a single line indicated in the height image. 
Left column of Figure 4 shows the topographic and ferroelectric state of the bilayer before poling. Both, PFM amplitude and phase shift, show no contrast along the whole area. This indicates a non-preferential orientation of the polymer dipoles in the as casted film. Right column of Figure 4 shows the topography and ferroelectric response of the polymer bilayer after poling in three selected positions. The topography image shows that in every case, the tip-surface interaction lead to indentation of the surface. Nonetheless, PFM amplitude and phase shift images show the development of ferroelectric contrast in the poled areas. The phase shift after poling is significantly smaller than the one observed in the hysteresis experiments (figure 3). This effect has been previously reported ${ }^{10-11,48}$ and has been related to the fact that the average vertical orientation of the dipole moments does not change significantly by the application of the electric field. It is known that $\mathrm{P}(\mathrm{VDF}-\mathrm{TrFE})$ crystals in thin films and in gratings are embedded in amorphous phase and they exhibit a needle like morphology which adopt a variety of orientations from flat-on to edge-on ${ }^{10}$. Therefore the value of the phase change can be attributed to the fact that the electric field is not applied over a single crystal but over a polycrystalline sample where crystals adopt different orientations. This fact makes phase change to be less than that expected for a single crystal.

In all three cases, the magnitude of the ferroelectric contrast seems to be comparable. However, the surface extension of the ferroelectric response varies from point to point. One might argue that this difference can be related to the indentation of the probe into the surface and the possible damage it may have caused to the ferroelectric top layer. The profile along the written spots, in the ferroelectric channels, show that the dipole orientation caused by the applied tip bias extends from 400 to $800 \mathrm{~nm}$. This result is in agreement with previous PFM studies in P(VDF-TrFE) SL thin films ${ }^{51}$. Considering the 
spreading of the ferroelectric information in the bilayer film, information storage density can be estimated as around $2 \pm 1 \mathrm{Gbit} / \mathrm{inch}^{2}$.

Figure 5 shows the LIPSS BL film, before and after poling at three spots, at $+12 \mathrm{~V}$ for 5 minutes. In this case, besides setting a low deflection cantilever set-point, the PFM study was carried out scanning the tip in a direction parallel to the LIPSS, thus possible damage due to tip-surface interaction was minimized ${ }^{10}$. Before poling, the left column of figure 5 shows that the irradiated structures have no preferential ferroelectric response. This indicates that the laser induced ripples do not align the P(VDF-TrFE) dipole moment in any preferential direction. However, it is interesting noticing that in the P(VDF-TrFE) droplets, on top of the LIPPS, the ferroelectric amplitude signal seems to have a lower value. This could serve as an indication of different crystallinity inside the droplets. The ferroelectric response of the LIPSS, after poling, is shown in the right column of figure 5. First, it is possible to observe that no change in the morphology of the nanostructures was generated by the tip interaction as evidenced in the topography image. Then, the PFM amplitude image shows the three written points on the LIPSS. The magnitude of the ferroelectric signal is comparable to the one in the continuous BL film. Nonetheless, the surface extension of the ferroelectric domains decreases significantly in comparison to the non-irradiated film. In the LIPSS, the electrical poling generates circular domains of about 100 to $200 \mathrm{~nm}$ in diameter, which correspond to an information density of $35 \pm 5 \mathrm{Gbit} / \mathrm{inch}^{2}$. It is worth mentioning the presence of some dots on the trench region. This is due to the nanometric control limitations of the piezoelectric scanner in the point and shoot protocol. Nevertheless, this proves that the material is also ferroelectric on the trench, further supporting the continuity of the upper polymer layer. The hindrance of the ferroelectric interaction in confined polymer structures, has been previously reported in nanogratings generated by 
nanoimprint lithography ${ }^{10-11,48}$ and attributed to the development of a higher proportion of edge-on lamellae, due to the confinement of the polymer crystals. Moreover, we have performed PFM measurements to test the dipole moment retention of the LIPSS bilayer. As presented in figure S4 (please refer to the supplementary information), four different points were poled at $+12 \mathrm{~V}$ (left side points) and $-12 \mathrm{~V}$ (right side points) for 5 minutes each. Then, we measured both the PFM amplitude and phase signals $18 \mathrm{~h}$ after poling. First, it is possible to affirm that, at laboratory times, the laser-structured bilayer retains the dipole information since both PFM signals did not show any quantitative variation with time. Second, we further highlight that poling with positive or negative bias do not strongly affect the absolute magnitude of the PFM amplitude signal (about 3-5 $\mathrm{mV})$, while the direction of both amplitude and phase show the characteristic behavior reported previously in the literature ${ }^{48}$.

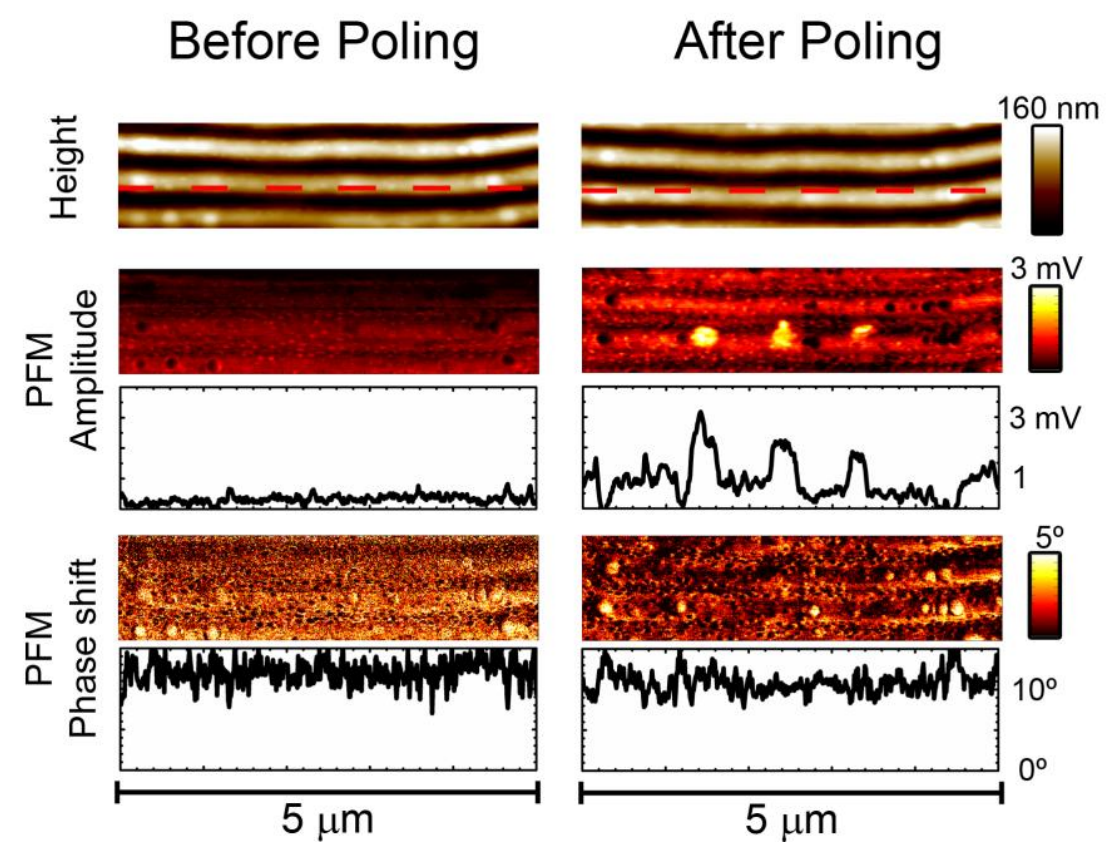

Figure 5. (Left column) Topography, PFM amplitude and PFM phase shift of the LIPSS on the bilayer film before poling. (Right column) Topography, PFM amplitude and PFM phase shift of the of the LIPSS of the bilayer film after poling at 3 points, for 5 minutes at $+12 \mathrm{~V}$. Panels third and fifth from top is the amplitude and phase shift across a single line indicated in the height image. 


\section{Conclusions}

Laser induced periodic surface structures can be prepared on polymer thin films, in spite of the absence of light absorption at the wavelength of the irradiating laser, by using a bilayer approach. We have shown that the structuring of a P3HT layer, by laser irradiation at $\lambda=532 \mathrm{~nm}$, covered by a ferroelectric $\mathrm{P}(\mathrm{VDF}-\mathrm{TrFE})$ thin layer allowed developing surface nanogratings. This approach will be useful not only for the case of $\mathrm{P}(\mathrm{VDF}-\mathrm{TrFE})$ but for any other non-absorbing polymer making the technique more versatile and thus paving the way for a wider range of applications. The LIPSS preserve the ferroelectric properties of the $\mathrm{P}(\mathrm{VDF}-\mathrm{TrFE})$, as evidenced by the hysteresis cycles recorded by PFM. The laser fabricated nanogratings showed an increase of the storage information density of about one order of magnitude, in comparison to the nonstructured bilayer.

\section{Acknowledgements}

The authors gratefully acknowledge the financial support of the Spanish Ministry of Economy and Competitiveness (MINECO) through MAT 2011-23455, MAT 201233517 and CTQ 2013-43086-P. D.E.M. thanks CSIC for the tenure of JAE-Pre fellowship and Fondo Social Europeo (FSE) for cofinancing the JAE Program. A. R. R. and E. R. thank MINECO for the tenure of a FPI contract (BES-2013-062620) and a Ramón y Cajal contract (RYC-2011-08069), respectively.

\section{References}

1. Asadi, K.; de Leeuw, D. M.; de Boer, B.; Blom, P. W. M., Organic Non-volatile Memories from Ferroelectric Phase-separated Blends. Nat Mater 2008, 7 (7), 547-550.

2. $\quad$ Nougaret, L.; Kassa, H. G.; Cai, R.; Patois, T.; Nysten, B.; van Breemen, A. J. J. M.; Gelinck, G. H.; de Leeuw, D. M.; Marrani, A.; Hu, Z.; Jonas, A. M., Nanoscale Design of 
Multifunctional Organic Layers for Low-Power High-Density Memory Devices. ACS Nano 2014, $8(4), 3498-3505$.

3. Naber, R. C. G.; de Boer, B.; Blom, P. W. M.; de Leeuw, D. M., Low-voltage Polymer Field-effect Transistors for Nonvolatile Memories. Applied Physics Letters 2005, 87 (20), 203509.

4. Cai, R.; Kassa, H. G.; Marrani, A.; van Breemen, A. J. J. M.; Gelinck, G. H.; Nysten, B.; Hu, Z.; Jonas, A. M., An Organic Ferroelectric Field Effect Transistor with Poly(vinylidene fluorideco-trifluoroethylene) Nanostripes as Gate Dielectric. Applied Physics Letters 2014, 105 (11), 113113.

5. Lee, K. H.; Lee, G.; Lee, K.; Oh, M. S.; Im, S.; Yoon, S.-M., High-Mobility Nonvolatile Memory Thin-Film Transistors with a Ferroelectric Polymer Interfacing ZnO and Pentacene Channels. Advanced Materials 2009, 21 (42), 4287-4291.

6. Bae, I.; Kim, R. H.; Hwang, S. K.; Kang, S. J.; Park, C., Laser-Induced Nondestructive Patterning of a Thin Ferroelectric Polymer Film with Controlled Crystals using Ge8Sb2Te11 Alloy Layer for Nonvolatile Memory. ACS Applied Materials \& Interfaces 2014, 6 (17), 1517115178.

7. Cauda, V.; Stassi, S.; Bejtka, K.; Canavese, G., Nanoconfinement: an Effective Way to Enhance PVDF Piezoelectric Properties. ACS Applied Materials \& Interfaces 2013, 5 (13), 6430 6437.

8. Cauda, V.; Torre, B.; Falqui, A.; Canavese, G.; Stassi, S.; Bein, T.; Pizzi, M., Confinement in Oriented Mesopores Induces Piezoelectric Behavior of Polymeric Nanowires. Chemistry of Materials 2012, 24 (21), 4215-4221.

9. $\quad$ Chen, X.-Z.; Li, Q.; Chen, X.; Guo, X.; Ge, H.-X.; Liu, Y.; Shen, Q.-D., Nano-Imprinted Ferroelectric Polymer Nanodot Arrays for High Density Data Storage. Advanced Functional Materials 2013, 23 (24), 3124-3129.

10. Martínez-Tong, D. E.; Soccio, M.; García-Gutiérrez, M. C.; Nogales, A.; Rueda, D. R.; Alayo, N.; Pérez-Murano, F.; Ezquerra, T. A., Improving Information Density in Ferroelectric Polymer Films by using Nanoimprinted Gratings. Applied Physics Letters 2013, 102 (19), 191601.

11. Kassa, H. G.; Cai, R.; Marrani, A.; Nysten, B.; Hu, Z.; Jonas, A. M., Structure and Ferroelectric Properties of Nanoimprinted Poly(vinylidene fluoride-ran-trifluoroethylene). Macromolecules 2013, 46 (21), 8569-8579.

12. Kang, S. J.; Bae, I.; Shin, Y. J.; Park, Y. J.; Huh, J.; Park, S.-M.; Kim, H.-C.; Park, C., Nonvolatile Polymer Memory with Nanoconfinement of Ferroelectric Crystals. Nano Letters 2010, 11 (1), 138-144.

13. Hisamoto, D.; Wen-Chin, L.; Kedzierski, J.; Takeuchi, H.; Asano, K.; Kuo, C.; Anderson, E.; Tsu-Jae, K.; Bokor, J.; Chenming, H., FinFET-a self-aligned double-gate MOSFET Scalable to $20 \mathrm{~nm}$. Electron Devices, IEEE Transactions on 2000, 47 (12), 2320-2325.

14. Tsai, H.; Pitera, J. W.; Miyazoe, H.; Bangsaruntip, S.; Engelmann, S. U.; Liu, C.-C.; Cheng, J. Y.; Bucchignano, J. J.; Klaus, D. P.; Joseph, E. A.; Sanders, D. P.; Colburn, M. E.; Guillorn, M. A., Two-Dimensional Pattern Formation Using Graphoepitaxy of PS-b-PMMA Block Copolymers for Advanced FinFET Device and Circuit Fabrication. ACS Nano 2014, 8 (5), 5227-5232.

15. Sevilla, G. A. T.; Rojas, J. P.; Fahad, H. M.; Hussain, A. M.; Ghanem, R.; Smith, C. E.; Hussain, M. M., Flexible and Transparent Silicon-on-Polymer Based Sub-20 nm Non-planar 3D FinFET for Brain-Architecture Inspired Computation. Advanced Materials 2014, 26 (18), 27942799.

16. Schift, H., Nanoimprint Lithography: An Old Story in Modern Times? A Review. Journal of Vacuum Science \&amp; Technology B 2008, 26 (2), 458-480.

17. Liu, Y.; Weiss, D. N.; Li, J., Rapid Nanoimprinting and Excellent Piezoresponse of Polymeric Ferroelectric Nanostructures. ACS Nano 2009, 4 (1), 83-90. 
18. Song, G.; Li, X.; Li, P.; Peng, Z.; She, X.; Li, J.; Sun, J., Assembly of One-dimensional Polymer Nanostructure Arrays by Photolithographic Approaches. Journal of Porous Materials 2012, 19 (2), 195-199.

19. Broglia, M. F.; Suarez, S.; Soldera, F.; Mücklich, F.; Barbero, C. A.; Bellingeri, R.; Alustiza, F.; Acevedo, D., Direct Laser Interference Patterning of Polystyrene Films Doped with azo dyes, using 355 nm Laser Light. Applied Surface Science 2014, 300, 86-90.

20. Yuan, D.; Lasagni, A.; Hendricks, J. L.; Martin, D. C.; Das, S., Patterning of Periodic Nanocavities on PEDOT-PSS using Nanosphere-Assisted Near-field Optical Enhancement and Laser Interference Lithography. Nanotechnology 2012, 23 (1), 015304.

21. Martín-Fabiani, I.; Siegel, J.; Riedel, S.; Boneberg, J.; Ezquerra, T. A.; Nogales, A., Nanostructuring Thin Polymer Films with Optical Near Fields. ACS Applied Materials \& Interfaces 2013, 5 (21), 11402-11408.

22. Rebollar, E.; Pérez, S.; Hernández, J. J.; Martín-Fabiani, I.; Rueda, D. R.; Ezquerra, T. A.; Castillejo, M., Assessment and Formation Mechanism of Laser-Induced Periodic Surface Structures on Polymer Spin-Coated Films in Real and Reciprocal Space. Langmuir 2011, 27 (9), 5596-5606.

23. Rebollar, E.; Vázquez de Aldana, J. R.; Pérez-Hernández, J. A.; Ezquerra, T. A.; Moreno, P.; Castillejo, M., Ultraviolet and Infrared Femtosecond Laser Induced Periodic Surface Structures on Thin Polymer Films. Applied Physics Letters 2012, 100 (4), 041106.

24. Castillejo, M.; Ezquerra, T. A.; Oujja, M.; Rebollar, E., Laser Nanofabrication of Soft Matter. In Lasers in Materials Science, Castillejo, M.; Ossi, P. M.; Zhigilei, L., Eds. Springer: Switzerland, 2014.

25. Guosheng, Z.; Fauchet, P. M.; Siegman, A. E., Growth of Spontaneous Periodic Surface Structures on Solids During Laser Illumination. Physical Review B 1982, 26 (10), 5366-5381.

26. Lu, Q.-h.; Wang, Z.-g.; Yin, J.; Zhu, Z.-k.; Hiraoka, H., Molecular Orientation in Laserinduced Periodic Microstructure on Polyimide Surface. Applied Physics Letters 2000, 76 (10), 1237-1239.

27. Varlamova, O.; Reif, J.; Varlamov, S.; Bestehorn, M., The Laser Polarization as Control Parameter in the Formation of Laser-induced Periodic Surface Structures: Comparison of Numerical and Experimental Results. Applied Surface Science 2011, 257 (12), 5465-5469.

28. Csete, M.; Marti, O.; Bor, Z., Laser-induced Periodic Surface Structures on Different Poly-carbonate Films. Applied Physics A 2001, 73 (4), 521-526.

29. Rebollar, E.; Perez, S.; Hernandez, M.; Domingo, C.; Martin, M.; Ezquerra, T. A.; GarciaRuiz, J. P.; Castillejo, M., Physicochemical Modifications Accompanying UV Laser Induced Surface Structures on Poly(ethylene terephthalate) and their Effect on Adhesion of Mesenchymal Cells. Physical Chemistry Chemical Physics 2014, 16 (33), 17551-17559.

30. Bäuerle, D. W., Laser Processing and Chemistry. Springer-Verlag: Berlin, 2011.

31. Bolle, M.; Lazare, S., Submicron periodic structures produced on polymer surfaces with polarized excimer laser ultraviolet radiation. Appl. Surf. Sci. 1993, 65-66, 349-354.

32. Naber, R. C. G.; Mulder, M.; de Boer, B.; Blom, P. W. M.; de Leeuw, D. M., High Charge Density and Mobility in poly(3-hexylthiophene) Using a Polarizable Gate Dielectric. Organic Electronics 2006, 7 (3), 132-136.

33. Naber, R. C. G.; Massolt, J.; Spijkman, M.; Asadi, K.; Blom, P. W. M.; de Leeuw, D. M., Origin of the Drain Current Bistability in Polymer Ferroelectric field-effect Transistors. Applied Physics Letters 2007, 90 (11), 113509.

34. Kim, Y.; Cook, S.; Tuladhar, S. M.; Choulis, S. A.; Nelson, J.; Durrant, J. R.; Bradley, D. D. C.; Giles, M.; McCulloch, I.; Ha, C.-S.; Ree, M., A Strong Regioregularity Effect in self-organizing Conjugated Polymer Films and high-efficiency polythiophene:fullerene Solar Cells. Nat Mater 2006, 5 (3), 197-203.

35. Chen, C.-P.; Chan, S.-H.; Chao, T.-C.; Ting, C.; Ko, B.-T., Low-Bandgap Poly(ThiophenePhenylene-Thiophene) Derivatives with Broaden Absorption Spectra for Use in High- 
Performance Bulk-Heterojunction Polymer Solar Cells. Journal of the American Chemical Society 2008, 130 (38), 12828-12833.

36. Palacios-Lidón, E.; Abellán, J.; Colchero, J.; Munuera, C.; Ocal, C., Quantitative Electrostatic Force Microscopy on Heterogeneous Nanoscale Samples. Applied Physics Letters 2005, 87 (15), 154106.

37. Riedel, C.; Arinero, R.; Tordjeman, P.; Lévêque, G.; Schwartz, G. A.; Alegria, A.; Colmenero, J., Nanodielectric Mapping of a Model polystyrene-poly(vinyl acetate) Blend by Electrostatic Force Microscopy. Physical Review E 2010, 81 (1), 010801.

38. Riedel, C.; Arinero, R.; Tordjeman, P.; Ramonda, M.; Lévêque, G.; Schwartz, G. A.; Oteyza, D. G. d.; Alegria, A.; Colmenero, J., Determination of the Nanoscale Dielectric Constant by means of a Double Pass Method using Electrostatic Force Microscopy. Journal of Applied Physics 2009, 106 (2), 024315.

39. de Silva, J. P.; Geoghegan, M.; Higgins, A. M.; Krausch, G.; David, M. O.; Reiter, G., Switching Layer Stability in a Polymer Bilayer by Thickness Variation. Physical Review Letters 2007, 98 (26), 267802.

40. Sharp, J. S.; Thomas, K. R.; Weir, M. P., Mechanically Driven Wrinkling Instability in Thin Film Polymer Bilayers. Physical Review E 2007, 75 (1), 011601.

41. Ade, H.; Winesett, D. A.; Smith, A. P.; Anders, S.; Stammler, T.; Heske, C.; Slep, D.; Rafailovich, M. H.; Sokolov, J.; Stöhr, J., Bulk and Surface Characterization of a Dewetting Thin Film Polymer Bilayer. Applied Physics Letters 1998, 73 (25), 3775-3777.

42. Xu, L.; Bandyopadhyay, D.; Shi, T.; An, L.; Sharma, A.; Joo, S. W., Dewetting Kinetics of Thin Polymer Bilayers: Role of Under Layer. Polymer 2011, 52 (19), 4345-4354.

43. Koo, J.; Shin, K.; Seo, Y.-S.; Koga, T.; Park, S.; Satija, S.; Chen, X.; Yoon, K.; Hsiao, B. S.; Sokolov, J. C.; Rafailovich, M. H., Stabilizing Thin Film Polymer Bilayers against Dewetting Using Multiwalled Carbon Nanotubes. Macromolecules 2007, 40 (26), 9510-9516.

44. Rodríguez-Rodríguez, Á.; Rebollar, E.; Soccio, M.; Ezquerra, T. A.; Rueda, D. R.; GarciaRamos, J. V.; Castillejo, M.; Garcia-Gutierrez, M.-C., Laser-Induced Periodic Surface Structures on Conjugated Polymers: Poly(3-hexylthiophene). Macromolecules 2015, 48 (12), 4024-4031. 45. Oujja, M.; Rebollar, E.; Abrusci, C.; Amo, A. D.; Catalina, F.; Castillejo, M., UV, Visible and IR Laser Interaction with Gelatine. Journal of Physics: Conference Series 2007, 59 (1), 571. 46. Hemmerlin, M.; Mermet, J. M., Determination of Elements in Polymers by Laser Ablation Inductively Coupled Plasma Atomic Emission Spectrometry: Effect of the Laser Beam Wavelength, Energy and Masking on the Ablation Threshold and Efficiency. Spectrochimica Acta Part B: Atomic Spectroscopy 1996, 51 (6), 579-589.

47. Zhao, Y.; Li, M.; Lu, Q., Surface Patterns Induced by Laser Irradiation on Thin Polymer Bilayer Films. Colloids and Surfaces A: Physicochemical and Engineering Aspects 2008, 312 (1), 47-55.

48. Hu, Z.; Tian, M.; Nysten, B.; Jonas, A. M., Regular Arrays of Highly Ordered Ferroelectric Polymer Nanostructures for Non-volatile Low-voltage Memories. Nat Mater 2009, 8 (1), 62-67. 49. Naber, R. C. G.; Asadi, K.; Blom, P. W. M.; de Leeuw, D. M.; de Boer, B., Organic Nonvolatile Memory Devices Based on Ferroelectricity. Advanced Materials 2010, 22 (9), 933945.

50. Park, C. H.; Lee, K. H.; Ha, T.; Kim, J. H.; Im, S.; Yun, J.; Lee, G. H., Ferroelectric Property Improvement of poly(vinylidene fluoride/trifluoroethylene) Polymer Exposed to a Plasma Ambient. Applied Physics Letters 2010, 97 (16), 162911.

51. Lee, J. S.; Prabu, A. A.; Kim, K. J., Annealing Effect upon Chain Orientation, Crystalline Morphology, and Polarizability of Ultra-thin P(VDF-TrFE) Film for Nonvolatile Polymer Memory Device. Polymer 2010, 51, 6319-6333.

52. Naber, R. C. G.; Asadi, K.; Blom, P. W. M.; de Leeuw, D. M.; de Boer, B., Organic Nonvolatile Memory Devices Based on Ferroelectricity. Advanced materials 2010, 22, 933-45. 
53. Bonnell, D. A.; Kalinin, S. V.; Kholkin, A. L.; Gruverman, A., Piezoresponse Force Microscopy: A Window into Electromechanical Behavior at the Nanoscale. MRS Bulletin 2011, 34, 648-657.

54. Kang, S. J.; Bae, I.; Shin, Y. J.; Park, Y. J.; Huh, J.; Park, S.-M.; Kim, H.-C.; Park, C., Nonvolatile Polymer Memory with Nanoconfinement of Ferroelectric Crystals. Nano letters 2011, 11, 138-44.

55. Oh, S.; Kim, Y.; Choi, Y.-Y.; Kim, D.; Choi, H.; No, K., Fabrication of Vertically WellAligned P(VDF-TrFE) Nanorod Arrays. Advanced Materials 2012, 5708-5712.

56. Zhu, L.; Wang, Q., Novel Ferroelectric Polymers for High Energy Density and Low Loss Dielectrics. Macromolecules 2012, 45, 2937-2954. 


\section{For table of contents use only}

\section{Laser Fabrication of Polymer Ferroelectric Nanostructures for Non-volatile Organic Memory Devices}

Daniel E. Martínez-Tong ${ }^{1, \neq *}$, Álvaro Rodríguez-Rodríguez ${ }^{1}$, Aurora Nogales ${ }^{1}$, Mari Cruz García-Gutiérrez ${ }^{1}$, Francesc Pérez-Murano ${ }^{2}$, Jordi Llobet ${ }^{2}$, Tiberio A. Ezquerra ${ }^{1}$ and Esther Rebollar,

${ }^{1}$ Instituto de Estructura de la Materia (IEM-CSIC). C/ Serrano 121. Madrid, 28006. Spain

${ }^{2}$ Instituto de Microelectrónica de Barcelona IMB-CNM (CSIC), Campus UAB 08193, Cerdanyola del Vallès (Bellaterra) Barcelona, Spain

${ }^{3}$ Instituto de Química Física Rocasolano (IQFR-CSIC). C/ Serrano 119. Madrid, 28006. Spain

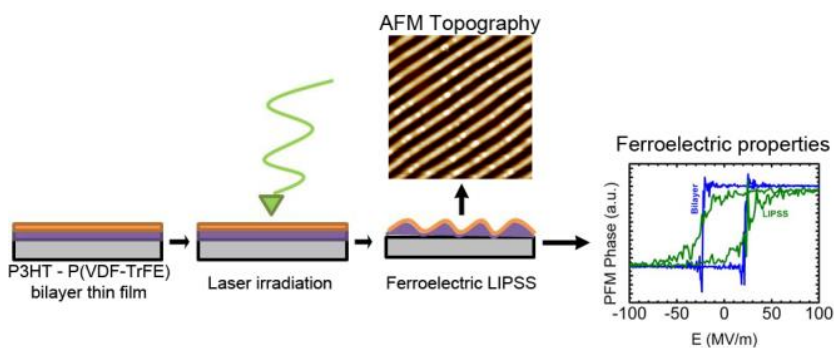

